\title{
Pattern Recognition: The Importance of Dispersion in Crystal Collimation
}

S. Shiraishi, University of Chicago

And

Steve Peggs, BNL

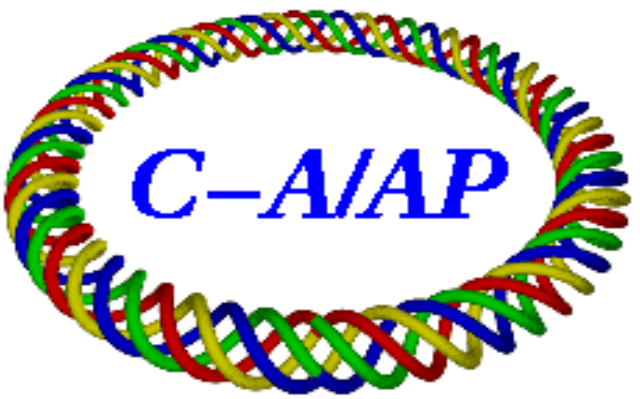

Collider-Accelerator Department

Brookhaven National Laboratory

Upton, NY 11973

Notice: This document has been authorized by employees of Brookhaven Science Associates, LLC under Contract No. DE-AC02-98CH10886 with the U.S. Department of Energy. The United States Government retains a nonexclusive, paid-up, irrevocable, world-wide license to publish or reproduce the published form of this document, or allow others to do so, for United States Government purposes. 


\title{
Pattern Recognition: \\ The Importance of Dispersion in Crystal Collimation
}

\author{
Satomi Shiraishi \\ University of Chicago \\ Steve Peggs \\ Brookhaven National Laboratory
}

\section{Introduction}

One aspect of the upcoming CRYSTAL experiment is to study the dynamics of single protons circulating the SPS in the presence of a crystal. Under some circumstances (for example under crystal channeling) a proton may hit the crystal and the neighboring silicon strip position detectors only once, before extraction from the SPS. In general (at most crystal rotation angles) it is expected that single protons will hit the crystal many times, with many accelerator turns between each hit, before escaping. Intermediate regimes are also possible (for example under volume reflection) in which a proton hits the crystal only a few times over many turns before being lost.

It is crucial that the data analysis of each single proton data set be able to distinguish between these different dynamical phases, and to be able to convincingly demonstrate that the fundamental processes at play in each phase are well understood. Distinguishing between dynamical phases depends crucially on the ability to perform pattern recognition - at least visually, but preferably quantitatively - on the single proton data sets.

This note shows that synchrotron oscillations significantly affect the hit pattern of a proton on the crystal. (By hit pattern we mean either the measurement vector of turn number and penetration depth, for each proton, or a vector that can be directly derived from the measurement vector, such as the vector of inferred synchrotron phase and penetration depth.) The analysis is (deliberately) as rudimentary as possible, using an elementary linear calculation which neither includes any higher order effects in the accelerator, nor any dynamical interactions between the test proton and the crystal or the silicon detectors.

Single particle simulation studies need to be carried out for CRYSTAL, exploring realistic effects besides dispersion, such as multiple scattering, dead zones, energy loss, dispersion slope, and linear coupling. Only after analysis software becomes available to interpret the output of such studies will it be possible to predict with any confidence that it will be possible to distinguish all single proton dynamical phases in the CRYSTAL experiment. Then reality will prevail. 


\section{Calculation Method}

To first order, the horizontal motion of a particle in an accelerator is described by the sum of its betatron and synchrotron oscillation displacements,

$$
x_{t o t} \equiv x_{\beta}+\eta \delta
$$

where $\eta$ is the dispersion, and betatron and synchrotron oscillations are:

$$
\begin{aligned}
x_{\beta} & =a_{x} \cos \left(\phi_{x}\right) \\
& =n_{x} \sigma_{x} \cos \left(2 \pi Q_{x} t+\theta_{x}\right), \\
\delta= & a_{s} \cos \left(\phi_{s}\right) \\
= & n_{s}\left(\sigma_{p} / p\right) \cos \left(2 \pi Q_{s} t+\theta_{s}\right) .
\end{aligned}
$$

The amplitudes and phases of the betatron and synchrotron oscillations are:

$$
\begin{aligned}
a_{x} & \equiv n_{x} \sigma_{x} \\
a_{s} & \equiv n_{s}\left(\sigma_{p} / p\right), \\
\phi_{x} & \equiv 2 \pi Q_{x} t+\theta_{x} \\
\phi_{s} & \equiv 2 \pi Q_{s} t+\theta_{s} .
\end{aligned}
$$

where $n_{x}$ and $n_{s}$ parametrize the amplitudes of the oscillations, and $\theta_{x}$ and $\theta_{s}$ are the initial phases. The beam size, $\sigma_{x}$, is calculated from the emittance, and the betatron and the synchrotron tunes are $Q_{x}$ and $Q_{s}$. Time is represented by the turn number, $t$.

The emittance and other SPS beam parameters used in this calculation are listed in Table 1. They are nominal values for CRYSTAL, except that the synchrotron tune deviates slightly from its nominal value of $Q_{s}=0.004$, for visual clarity below. Note the the dispersion at the crystal has a large magnitude, and is negative.

The betatron and synchrotron oscillation range of a test particle at a crystal with negative dispersion is indicated by the black rectangle in Fig. 1. The crystal displacement

$$
\begin{aligned}
x_{c} & \equiv n_{c} \sigma_{x} \\
& =6 \sigma_{x},
\end{aligned}
$$

is indicated with a solid blue line in Fig. 1. If $\delta=0$, the particle moves on the horizontal axis and hits the crystal only if the betatron oscillation amplitude, $a_{x}$, is greater than $x_{c}$. However, if $\delta \neq 0$, the particle can hit the crystal even if $a_{x}$ is less than $x_{c}$. Similarly, if $\delta$ is large, a particle can miss the crystal even if $a_{x}$ is greater than $x_{c}$ because the dispersion is negative. The hit pattern of a particle is altered when significant dispersion is present. 


\begin{tabular}{|l|c|}
\hline Property & Values \\
\hline Betatron tune, $Q_{x}$ & 26.135 \\
Synchrotron tune, $Q_{s}$ & 0.0045 \\
Dispersion at crystal, $\eta[\mathrm{m}]$ & -0.88 \\
Beta function at crystal, $\beta_{x}[\mathrm{~m}]$ & 96.05 \\
Normalized emittance, $\epsilon[\mathrm{rad} \mathrm{m}]$ & $1.5 \times 10^{-6}$ \\
Beam size, $\sigma_{x}[\mathrm{~m}]$ & $1.06 \times 10^{-3}$ \\
Momentum, $p[\mathrm{GeV} / c]$ & 120 \\
$\mathrm{RMS} \Delta p / p,\left(\sigma_{p} / p\right)$ & $4 \times 10^{-4}$ \\
$|\eta|\left(\sigma_{p} / p\right)[\mathrm{m}]$ & $0.35 \times 10^{-3}$ \\
Crystal edge displacement, $x_{c}$ & $6 \sigma_{x}$ \\
\hline
\end{tabular}

Table 1: Nominal SPS Lattice and beam parameters used for the calculation. Note that the dispersion is negative and has a large magnitude.

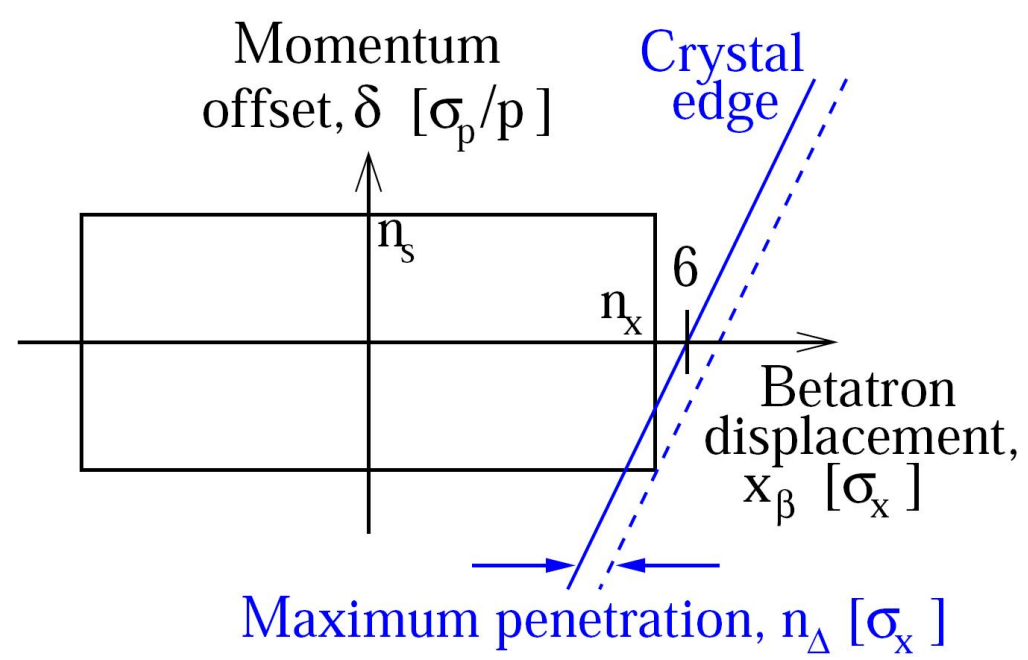

Figure 1: Momentum offset and betatron displacement for a particle. The location of the crystal edge $\left(n_{c}=6\right)$ is indicated with a solid blue line. The maximum penetration depth $\Delta=n_{\Delta} \sigma_{x}$ is indicated with a dashed blue line. 


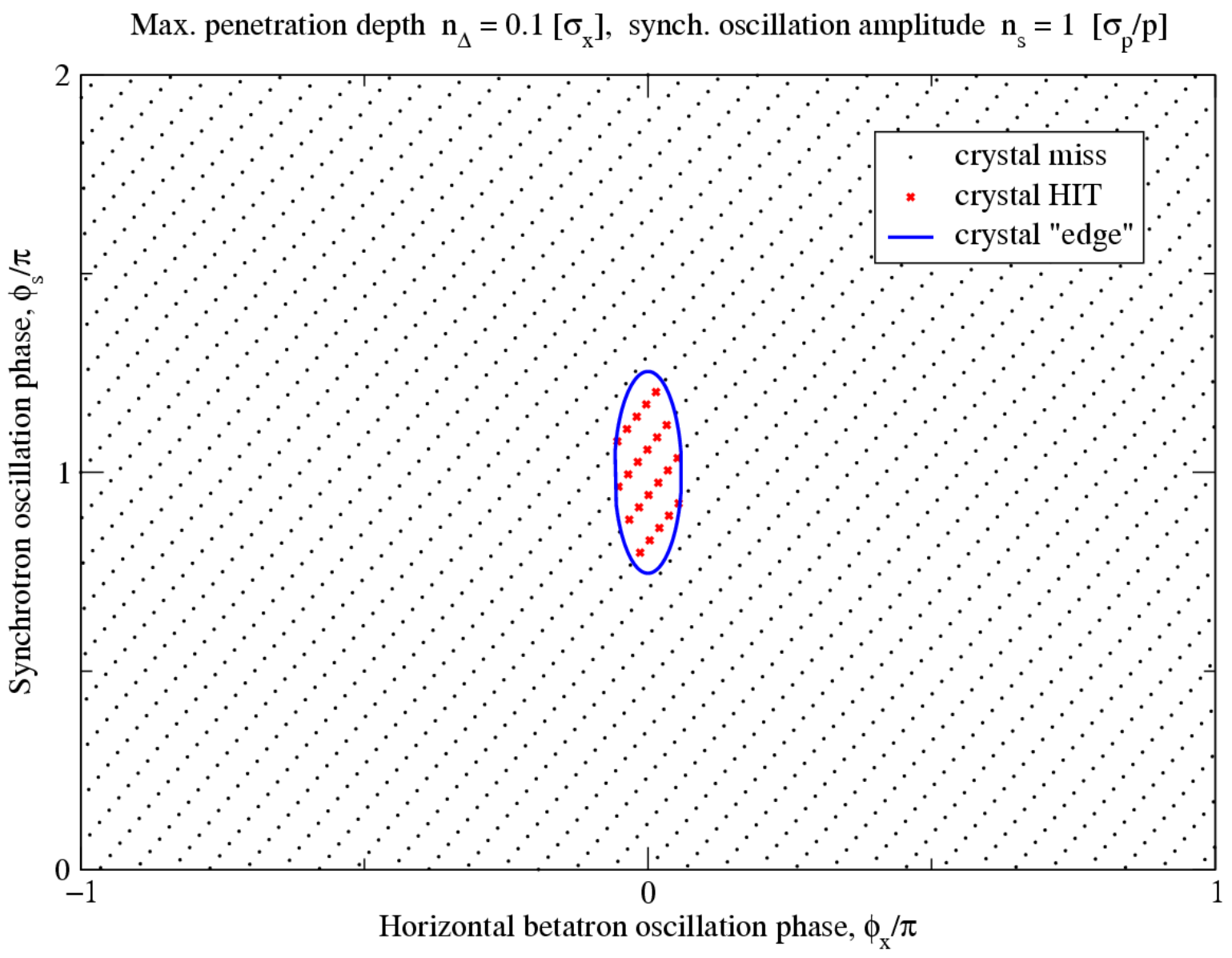

Figure 2: The betatron and synchrotron oscillation phases for 2,000 turns with $n_{s}=1$ and $n_{\Delta}=0.1$. The phases that do not hit the crystal are dots and the phases that hit the crystal are small red crosses. The edge of the crystal is indicated with a blue line.

To investigate the hit pattern of a single particle, we calculate the phases and the horizontal positions of that particle during successive turns for various synchrotron oscillation amplitudes and maximum penetration depths in the crystal. Particle interactions with the crystal or the detector are not included in the calculation. We define a parameter, $n_{\Delta}$, for the maximum penetration, $\Delta$ :

$$
\Delta \equiv n_{\Delta} \sigma_{x}
$$

Since the maximum penetration into the crystal occurs when the betatron oscillations and the synchrotron oscillations are at their maxima, the oscillation amplitudes and the crystal location are related as follows:

$$
\begin{aligned}
a_{x}+|\eta| a_{s} & =x_{c}+\Delta \\
n_{x} \sigma_{x}+|\eta| n_{s}\left(\sigma_{p} / p\right) & =n_{c} \sigma_{x}+n_{\Delta} \sigma_{x} .
\end{aligned}
$$


We vary $n_{s}$ to change the synchrotron oscillation amplitude and $n_{\Delta}$ to vary the maximum penetration depth in order to identify which combinations of oscillation phases hit the crystal. The phases of a particle with $n_{s}=1$ and $n_{\Delta}=0.1(\Delta=106 \mu \mathrm{m})$ are plotted in Fig. 2, which shows that a particle only rarely hits the crystal when significant dispersion is present.

A particle hits the crystal at a different rate when the dispersion is significant. This changes the hit pattern for a particle. It is necessary to include the effect of dispersion in order to identify a single particle by its hit pattern, and in order to derive its synchrotron and betatron amplitudes. 


\section{Dependence of hit pattern on penetration depth}

Phases at the crystal edge are shown in Fig. 3 for a fixed synchrotron oscillation amplitude of $a_{s}=1\left(\sigma_{p} / p\right)$ for a set of maximum penetration depths $n_{\Delta}$. The crystal edge encompasses a larger range of phases when the maximum penetration depth is larger, indicating that a particle will hit the crystal more frequently.

Synchrotron oscillation amplitude $\mathrm{n}_{\mathrm{s}}=1.0[\sigma / \mathrm{p}]$

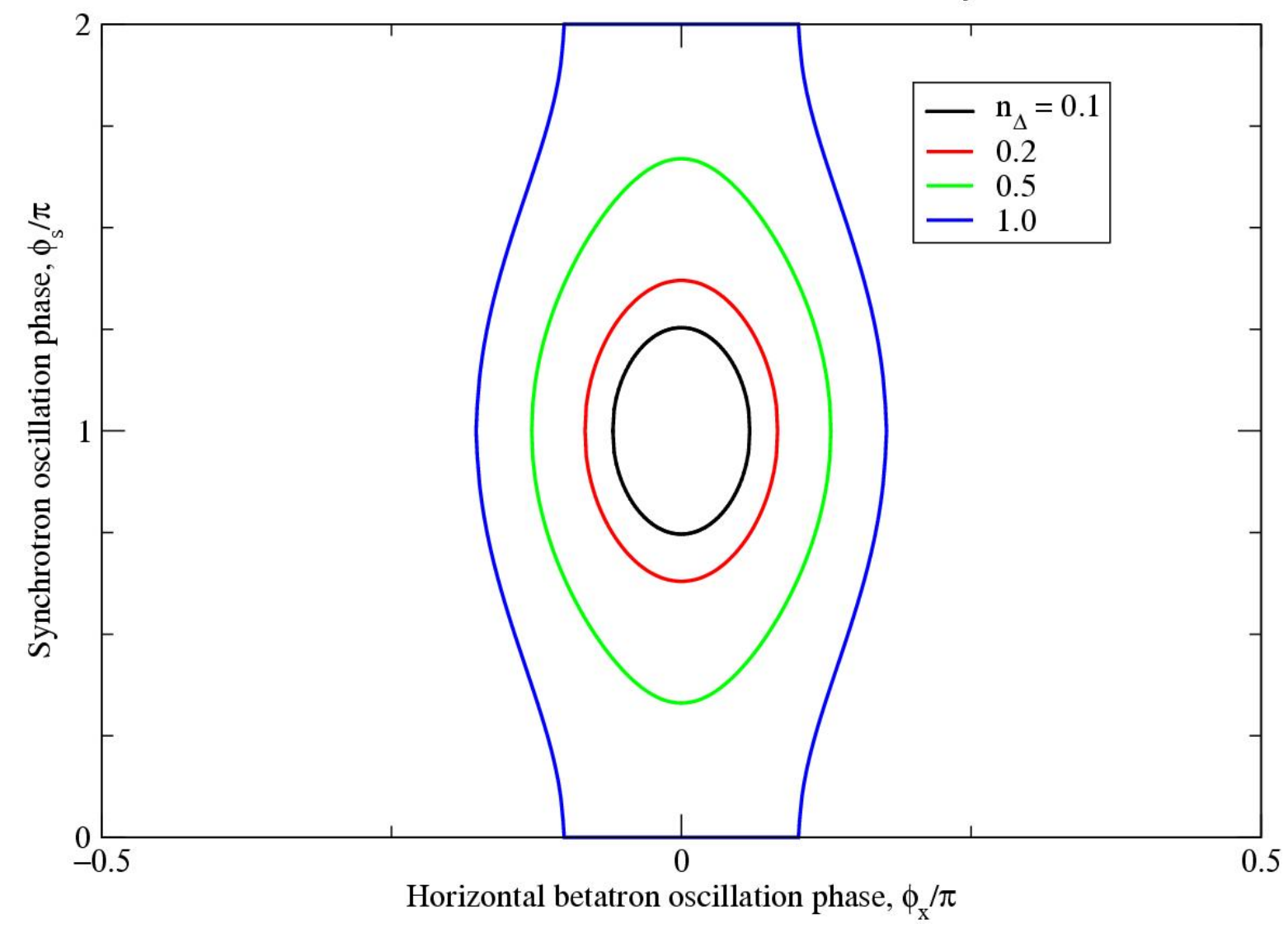

Figure 3: Phases of the crystal edge for a fixed synchrotron oscillation amplitude of $1\left(\sigma_{p} / p\right)$ with maximum penetration depths of $n_{\Delta}=0.1,0.2,0.5$, and 1.0. Note that the betatron phase runs only from $-\pi / 2 \leq \phi_{x} \leq \pi / 2$.

Figure 4 shows the hit pattern as a function of time. Points are plotted at the maximum penetration depth, $\Delta=n_{\Delta} \sigma_{x}$, when the particle hits the crystal. When $\Delta$ is large (ex. $n_{\Delta}=1$ ), the particle "continuously" hits the crystal. When $\Delta$ is small (ex. $n_{\Delta}=0.1$ ), the particle only hits two or three times per synchrotron period. For a given synchrotron oscillation amplitude, this difference in the hit pattern is a signature to identify each individual particle, and its maximum penetration depth. 
Synchrotron oscillation amplitude $n_{s}=1\left[\sigma_{p} / p\right]$

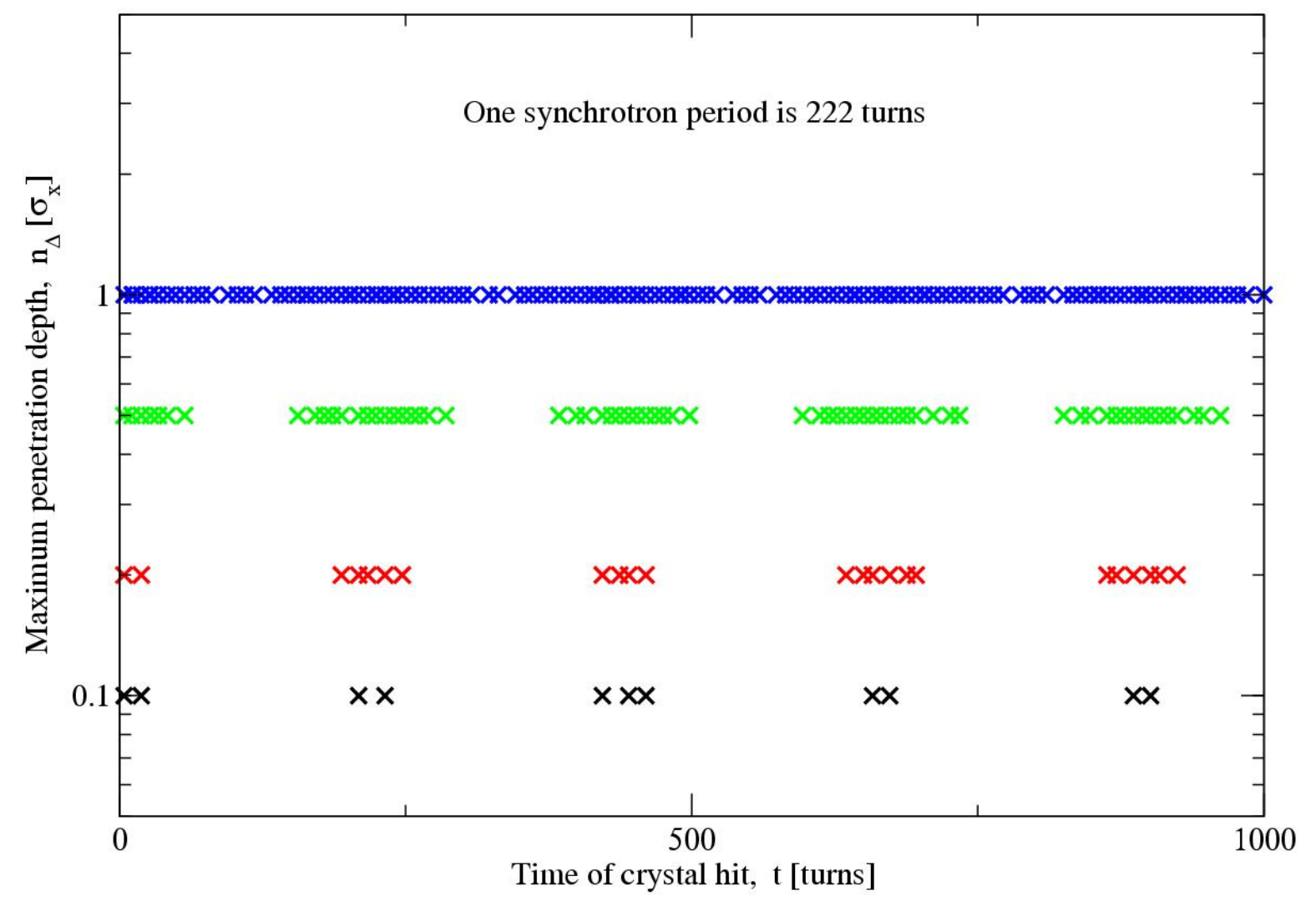

Figure 4: Hit patterns for maximum penetration depths $n_{\Delta}=0.1,0.2,0.5$, and 1.0. Crosses indicate turns in which a particle hits the crystal. 


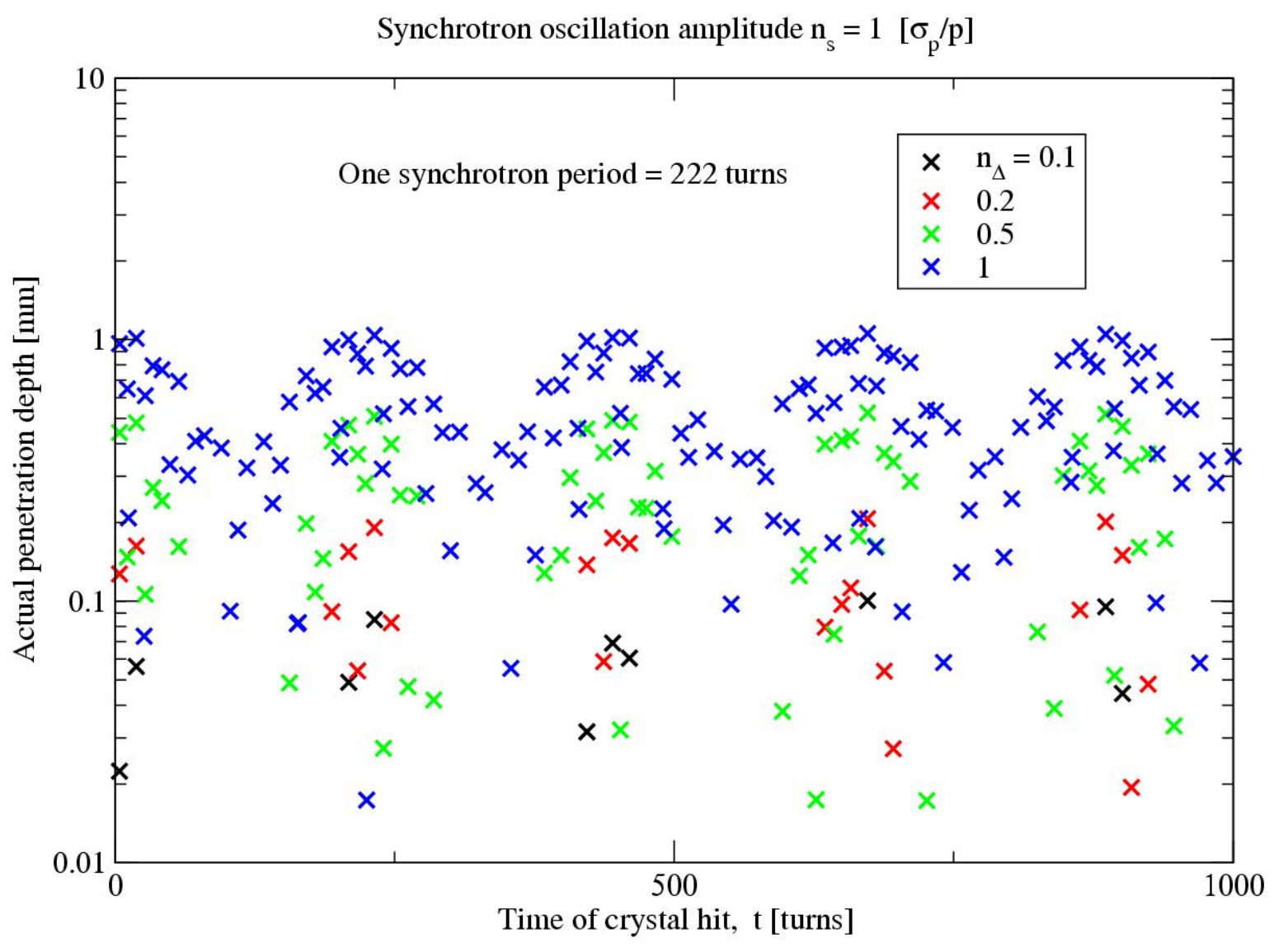

Figure 5: Actual penetration depths as a function of time for various maximum penetration depths $n_{\Delta}=0.1,0.2,0.5$, and 1.0.

Figure 5 shows the actual penetration depth as a function of time. Hit pattern is clearly periodic with the synchrotron oscillation period, but the identification of individual particles from the pattern is difficult.

Figure 6 shows the actual penetration depth as a function of synchrotron phase in polar coordinates. It illustrates distinct profiles for each penetration depth pattern more clearly than Fig. 5. Different maximum penetration depths are color coded to indicate the scale of each profile. The size and the shape of the profile is also a signature to identify each individual particle.

Realistic effects like multiple scattering and energy losses (in the crystal, detectors, and Roman Pot walls) will change these distinctive profiles. For example, a "dead zone" at the edge of a silicon detector (perhaps due to the edge of a Roman Pot) would appear as a hole in the center. Since a particle often hits at small penetration depth, the presence of a dead zone implies that we may not be able to identify many particles. 
Synchrotron oscillation amplitude $n_{\mathrm{s}}=1\left[\sigma_{\mathrm{p}} / \mathrm{p}\right]$

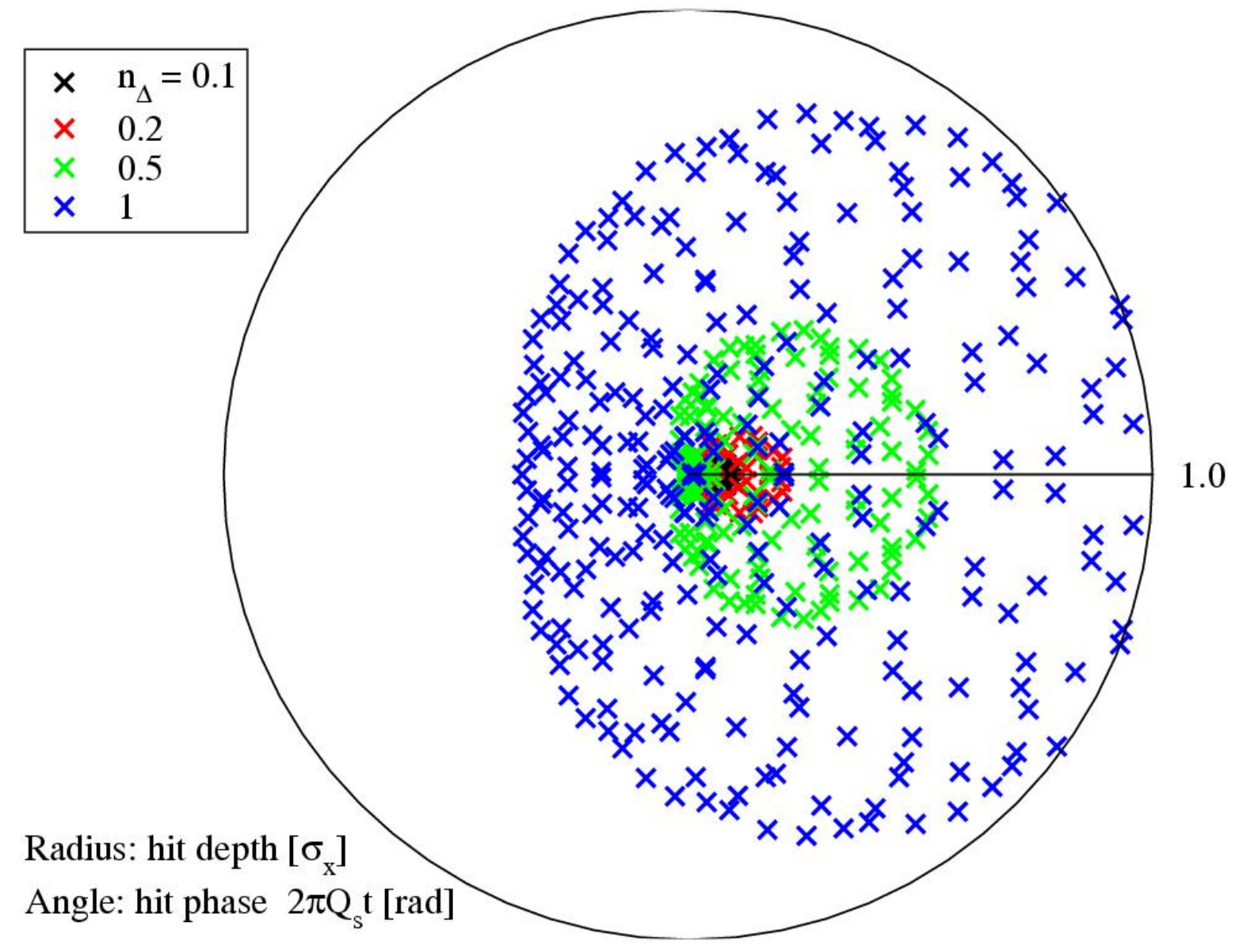

Figure 6: Penetration depths as a function of inferred synchrotron phase for various maximum penetration depths $n_{\Delta}=0.1,0.2,0.5$, and 1.0 . 


\section{Dependence on synchrotron oscillation amplitude}

In the previous section, we fixed the synchrotron oscillation amplitude and varied the maximum penetration depth. Here, we fix the maximum penetration depth to $n_{\Delta}=$ $0.1(\Delta=106 \mu \mathrm{m})$ and vary the synchrotron oscillation amplitude. Figure 7 shows the crystal edges for various synchrotron oscillation amplitudes. As the amplitude increases the available range of phases that allow a particle to hit the crystal becomes increasingly limited. This implies that a particle with a large synchrotron oscillation amplitude rarely hits the crystal.

Maximum penetration depth $\mathrm{n}_{\Delta}=0.1\left[\sigma_{\mathrm{x}}\right]$

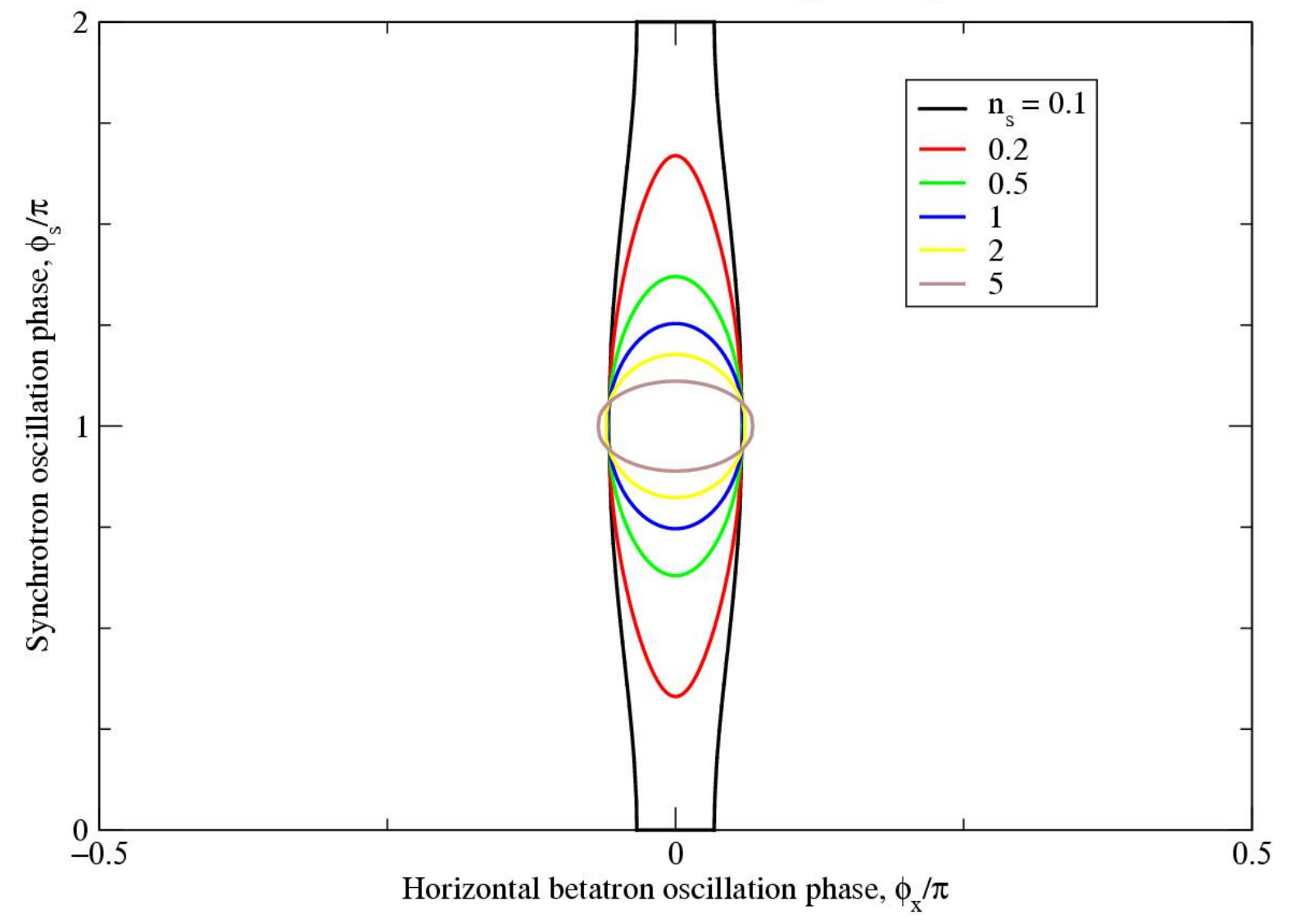

Figure 7: Phases of the crystal edge for synchrotron oscillation amplitudes $n_{s}=0.1$, $0.2,0.5,1,2$, and 5 . The betatron phase extends only from $-\pi / 2 \leq \phi_{x} \leq \pi / 2$

The hit pattern as a function of time is shown in Fig. 8. A point is drawn at its synchrotron oscillation amplitude for each turn in which a particle hits the crystal. For a fixed maximum penetration depth, a small synchrotron oscillation amplitude leads to frequent hits, but hits become less frequent as the amplitude increases. The hit pattern is periodic with a synchrotron oscillation period of about 222 turns. Similar to 
the case of varying maximum penetration depths, this hit pattern is also a signature to identify particles with different synchrotron oscillation amplitudes.

The hit depth as a function of time is shown in Fig. 9. Distinction of synchrotron oscillation amplitudes from the hit patterns are difficult from this figure.

Figure 10 shows the hit depth as a function of the inferred synchrotron phase in polar coordinates. Each hit pattern shows a clear profile whose size and shape depends on the synchrotron oscillation amplitude.

Again, any dead zone in the detector would appear as a hole in the center of the plot, so a large fraction of particle hits could be missed.

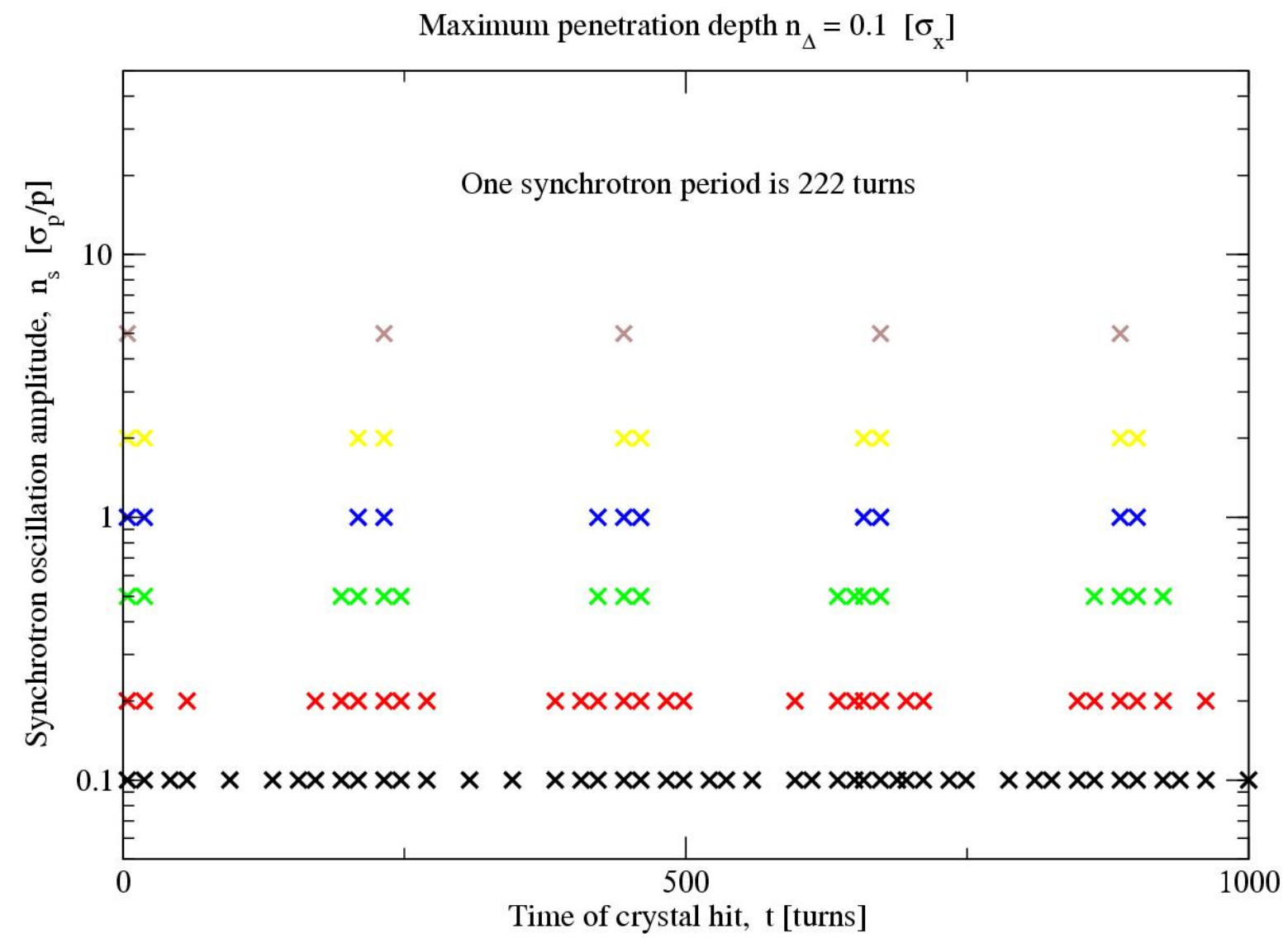

Figure 8: Hit patterns for maximum penetration depths $n_{\Delta}=0.1,0.2,0.5$, and 1.0. Points indicate turns in which a particle hits the crystal. 


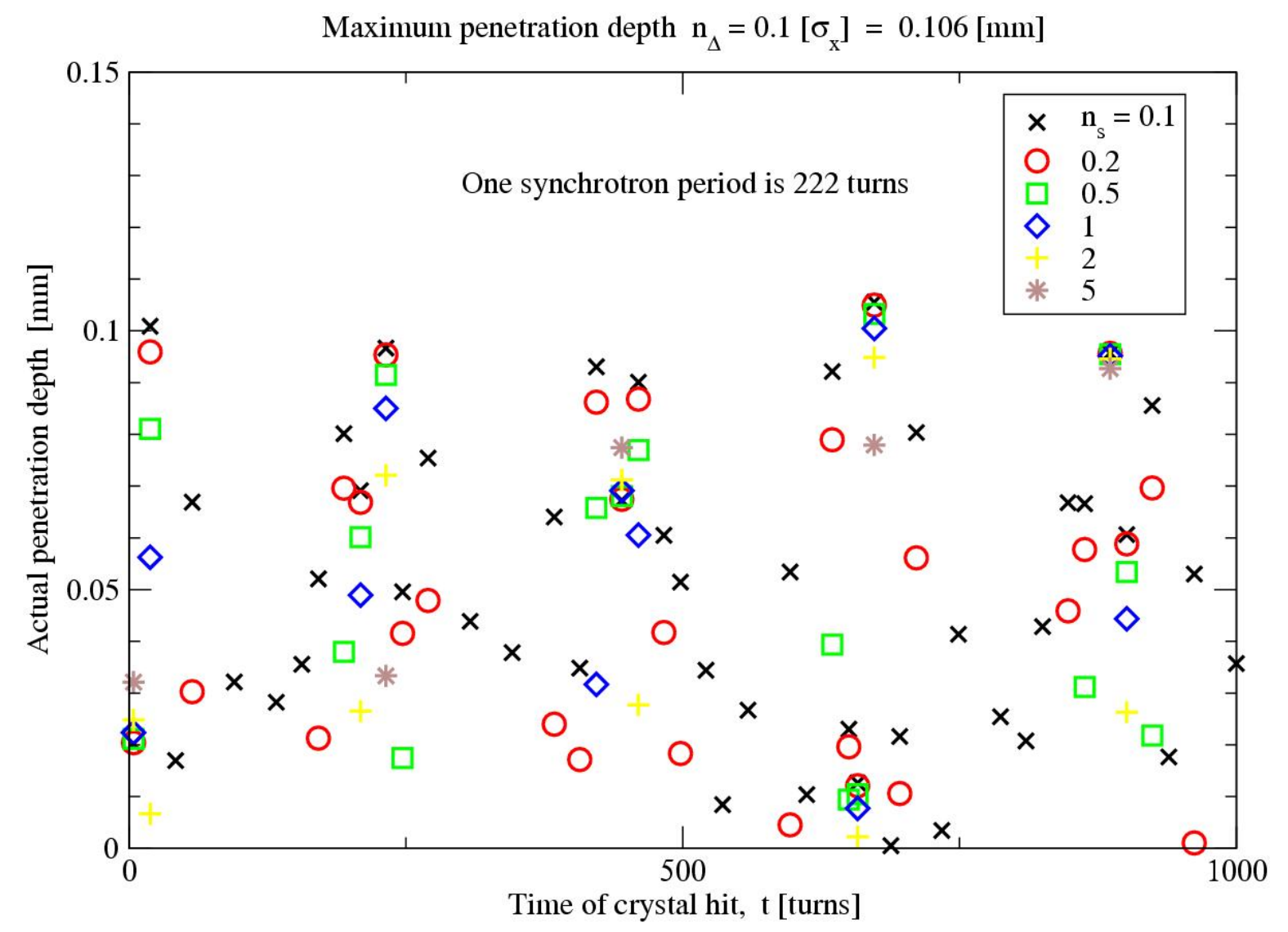

Figure 9: Actual penetration depths as a function of time for synchrotron oscillation amplitudes of $n_{s}=0.1,0.2,0.5,1,2$, and 5 . The maximum penetration depth is fixed to $n_{\Delta}=0.1$. 
Maximum penetration depth $\mathrm{n}_{\Delta}=0.1\left[\sigma_{\mathrm{x}}\right]$

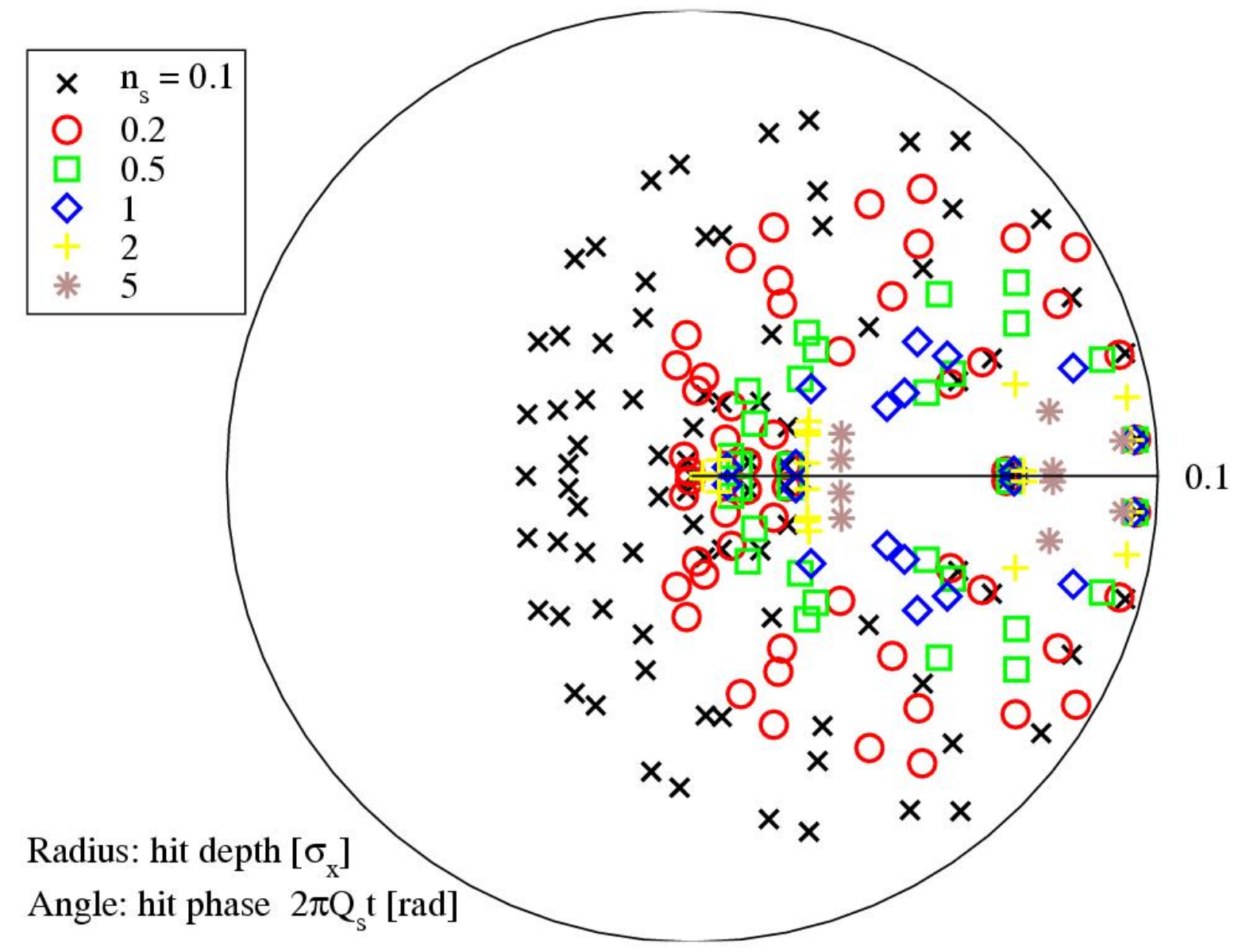

Figure 10: Actual penetration depths as a function of inferred synchrotron oscillation phase for synchrotron oscillation amplitudes of $n_{s}=0.1,0.2,0.5,1,2$, and 5 . The maximum penetration depth is fixed as $n_{\Delta}=0.1$. 


\section{Acknowledgments}

We would like to acknowledge the input and encouragement of Angelika Drees, YoungKee Kim, Michela Prest, Guillaume Robert-Demolaize, and Walter Scandale.

\section{Conclusions}

The presence of significant dispersion will have a strong effect on our ability to distinguish between single particle dynamical phases during the analysis of data from the CRYSTAL experiment in the SPS. In particular, the hit pattern depends on both maximum penetration depth and on synchrotron oscillation amplitude. Proper consideration of dispersion in the experiment is crucial, in both simulation and analysis.

Plots of the measured penetration depth as a function of inferred synchrotron phase are strong candidates for use in pattern recognition to identify individual particles, to quantitatively deduce their oscillation amplitudes and phases, and to distinguish their dynamical phases. Plots in polar co-ordinates are especially visually striking.

Protons hitting the crystal with a small penetration depth - in the dead zone of the Roman Pot(s) - will be unobserved. The large variation of the actual penetration depth with synchrotron phase shows that such dead zones will make the identification and interpretation of individual protons more difficult, especially (but not only) for protons with small maximum penetration depths. Thus, the presence of a "near detector" immediately adjacent to the crystal, with the smallest possible dead zone, is highly desirable.

Other single particle effects also need study, such as multiple scattering, energy loss, dead zones, dispersion slope and linear coupling. They need to be included in the generation of simulated single proton data sets. Analysis software should be capable of diagnosing the presence and importance of such effects. 